\title{
La teoría de la justicia social en Rawls. ¿Suficiente para enfrentar las consecuencias del capitalismo?
}

\author{
Paula Francisca Vidal Molina*
}

Resumen: En este artículo se presenta un abordaje general de la teoría de la justicia social de John Rawls. Revisamos algunas críticas desde diversos frentes teóricos. Consideramos que la justicia social permite enjuiciar las consecuencias regresivas para la humanidad del capitalismo en su fase neoliberal y comenzar a imaginar otros mundos posibles.

Palabras clave: Justicia social, capitalismo, consecuencias, Rawls.

\section{The theory of social justice in Rawls. Is it enough to confront the consequences of capitalism?}

\begin{abstract}
On this essay is presented a general approach to the theory of social justice in John Rawls. We review some critic views coming from several theoretical approaches. We believe that social justice allows us to judge the regressive consequences for humanity of capitalism in its neoliberal phase and to imagine other possible worlds.
\end{abstract}

Key words: Social justice, capitalism, impacts, Rawls.

Recibido: 20.05.2009

Aceptado: 01.07.2009

$$
* * *
$$

\section{Algunos Supuestos}

Algunos dirán que además de volver cada cierto tiempo como una moda que renueva líneas clásicas, permite justificar acciones que en nada cambian el panorama actual; la semántica de la justicia aparece cual sombra de infinitos movimientos. Un sin número de instancias contradictorias y análogas ocupan, usan, apelan al concepto de justicia, sin embargo, lo que diferencia una de otra es el modo como conciben y materializan ese concepto.

\footnotetext{
* Universidad Federal de Río de Janeiro, Río de Janeiro, Brasil. E-mail: pvidal71yahoo.com
} 
La relevancia de visibilizar y profundizar algunos elementos desdibujados, pero que se conjugan a la hora de hablar de justicia social, está lejos de constituirse en un mero ejercicio estético, de erudición, de lógica o moda, más bien nos enfrenta a la vitalidad de la pregunta por el tipo de sociedad en la que deseamos vivir y queremos construir para futuras generaciones. La pregunta -instalada aparentemente en la carga genética de la humanidad y capaz de gestar los más grandes y sublimes movimientos de lo humano como también los más miserables y vacíos- nos enfrenta con nuestra cotidianidad y el futuro saturado de pura posibilidad.

Justicia es una palabra con una carga teórica e histórica que esconde discursos y prácticas que los afirman, combaten o tensionan. Hoy se teje junto a otras como ciudadanía, democracia, bienestar, comunidad, reconocimiento, derechos, equidad, etc., todo lo cual configura una trama colmada de contradicciones y pugnas, que se ubican muy lejos de la aparente asepsia del mundo conceptual. Aquí esa trama de conceptos no será objeto de estudio, solo se asumen como sustrato y consecuencias de la discusión sobre la justicia social.

Fijar la mirada en un concepto permite leer la multiplicidad de sentidos que se atribuyen -en este caso- a la noción de justicia y la extraordinaria confusión que provoca su uso. Perelman ya anunciaba que las concepciones más corrientes la significan como: a cada cual la misma cosa, a cada cual según sus méritos, a cada cual según sus obras, a cada cual según sus necesidades, a cada cual según su posición o a cada cual según lo que la ley le atribuye (Perelman, Chaïn; 2005).

Cada uso es puesto en cuestión si se observa a la luz de los fenómenos regresivos de la sociedad contemporánea, donde el creciente aumento de la pobreza, la permanente violación de los derechos sociales, económicos, políticos, culturales y ambientales, el aumento de la desigualdad entre países y al interior de estos, es parte del paisaje que viven millones de personas.

En este marco, nos parece imprescindible conocer, analizar y tensionar a la luz de esos fenómenos una de las teorías contemporáneas de la justicia -la de Rawls- porque ha instalado renovadamente el concepto de justicia social e influenciado nuevas y viejas reflexiones al respecto, especialmente dentro del marco del Estado Liberal. Si bien, la obra de este autor nace y se inscribe en países y debates de los países centrales, creemos que su producción viene influenciando también las preocupaciones dentro y fuera del mundo académico en América Latina, ya sea a través de las exigencias y "recomendaciones" de lineamientos internacionales para con la semántica y el hacer de las políticas públicas, o con los debates hegemónicos del mundo académico internacional -especialmente de los países centrales- cuyo peso alcanza más allá de su órbita. Por lo mismo se requiere conocer con mayor rigurosidad los planteamientos con el fin de evaluar su pertinencia en el momento actual de la sociedad contemporánea. 
A Rawls algunos autores lo ubican como un promotor de un Estado liberal-socialdemócrata, o liberal igualitario donde el Estado asume una función activa de preocupación por las necesidades de la sociedad, por lo mismo se lo tilda de progresista en relación a la tradición de Nozick o de la vertiente "anarco-liberal".

El telón de fondo desde donde construye su sistema teórico es la legitimidad que posee el sistema económico de libre mercado y el Estado de Derecho, por lo tanto, conocer profundamente esta versión, permite elaborar críticas de fondo que apunten a la superación de ese orden. En ese contexto, parece pertinente concebir la propuestas de justicia en analogía con lo que en música llaman el sistema tonal, donde la base que domina es el capitalismo, una economía de libre mercado, y tanto la estructura institucional como otras esferas de la vida social se subordinan a sus requerimientos o la tensionan sin apuntar a modificar lo fundamental. En función de ello, parece urgente visualizar -siguiendo a Callinicos- otras vías que se levanten como alternativa teórico filosófico al modelo liberal (y sus variantes) en las discusiones sobre la justicia, ya que (re)construir un horizonte normativo es parte del proceso de concebir, imaginar un modelo de sociedad anticapitalista que guíe las diversas acciones a emprender.

\section{John Rawls y la Teoría de la Justicia}

Previo a explicitar los argumentos que constituyen la "Teoría de la Justicia" de Rawls, debemos señalar que con esta obra, salida a luz en 1971, se propone como objetivo ofrecer, en el contexto de la filosofía moral moderna una concepción moral alternativa a la derivada del utilitarismo clásico, el perfeccionismo y la opción intuicionista.

Este texto para muchos teóricos, discípulos y oponentes de Rawls, ha significado atribuirle la revitalización de la filosofía moral que en esa época estaba en peligro de constituirse en algo estéril producto del énfasis en análisis lógicos y semánticos de conceptos éticos y metaéticos, todo lo cual había desviado la atención de cuestiones prácticas y materiales (Kukathas y Pettit, 1990; Van Parijs, 1992; Gargarella, 1999; Borón, 2003; Massini, 2005; entre otros). Lo anterior unido a la agitación social y política de la época, los cuestionamientos morales de la guerra de Vietnam, el papel decadente que venía teniendo el positivismo analítico en la década de los setenta, tradición que había dejado de lado la cuestión de la justicia, vienen a sumarse para que "Teoría de la Justicia” se inserte en ese proceso de superación del positivismo analítico y adquiera la dimensión de un clásico. Otros, también le asignan a él ser el precursor de la mayoría de edad de la filosofía política liberal, en tanto incorpora en la tradición individualista la idea de que las sociedades tienen pautas de desigualdad que persisten en el tiempo y a la vez, modos sistemáticos mediante las cuales se asigna a las personas posiciones dentro de jerarquías de poder, estatus y dinero (Barry, en Callinicos; 2003). Vallespín (1985) agrega que la salida a luz de la Teoría de la Justicia es oportuna debido a la crisis de legitimidad que 
cruzaba a la teoría democrática clásica en ese momento y la necesidad que se tenía de renovar las bases del liberalismo para permitir orientar y enjuiciar las prácticas institucionales.

\section{La Justicia como Primera Virtud de las Instituciones Sociales}

Rawls le otorga prioridad absoluta a la justicia y la entiende como la primera virtud de las instituciones sociales, por lo mismo en su planteamiento no le concede un valor mayor a las instituciones por presentarse ordenas, estables o eficientes, lo fundamental -menciona- será que ellas sean justas y en el caso de no serlo deberán atenerse a ser reformadas o invalidadas.

Con ello deja claro que su punto de partida y el foco de la justicia social es la estructura básica de la sociedad y en ello especialmente el modo en que las instituciones sociales distribuyen deberes y derechos al interior de la sociedad. Reconoce que a través de ellas se pueden favorecer o no ciertas posiciones iniciales en que se encuentran los sujetos en la sociedad, o crear desigualdades entre las personas -por cierto no justificadas en el mérito o el desmerecimiento- que indudablemente influirán en las expectativas de vida que tengan. Algunas de estas instituciones que Rawls menciona son la familia monogámica, las leyes, la constitución política, la protección jurídica de libre pensamiento y de la propiedad privada de los medios de producción, entre otros. Su propuesta concibe una idea de sociedad definida como una asociación de personas que reconocen ciertas reglas o pautas públicas de conducta, de orden obligatorias que permiten relacionarse y actuar. Estima que tales normas especifican un cierto sistema de cooperación que permite promover el bienestar de aquellos que participan del.

La cooperación en sociedad -en el modelo rawlsiano que parece retomar de los clásicos liberales- se caracteriza por estar constituido por dos polos; la identidad y el conflicto de intereses. La identidad de intereses deja ver la concurrencia de todos para permitir una vida mejor que aquella posible de obtener según el esfuerzo particular e aislado de cada persona. No obstante -refiere- que el conflicto de intereses es constitutivo al interior de un grupo, debido a que las personas no son indiferentes acerca de la forma de distribuir los mayores beneficios que resultan de la colaboración entre las personas, siempre ellas van a preferir tener una mayor participación en función de la obtención de sus fines.

Bajo estos supuestos, afirma la necesidad de contar con un conjunto de principios de justicia que permitan definir el modo de asignar derechos y deberes por parte de las instituciones básicas y con ello, definir la distribución "apropiada de los beneficios y las cargas de cooperación social”. De esta manera, una concepción de justicia otorga un criterio para evaluar los aspectos distributivos que permite la estructura básica de la 
sociedad, principios de justicia que permiten regular, por ejemplo la constitución política, instancias del Estado y elementos del sistema económico y social. Pero ello implica -en este modelo- asumir un sentido público de la justicia y una concepción compartida de ella, donde las diferencias de intereses y objetivos de las personas, no afecte establecer vínculos de "amistad cívica”.

Admite la existencia de diferencias de facto entre las personas acerca de lo que estiman justo o injusto en la sociedad, pero este reconocimiento no anula que cada uno posea una concepción de justicia y a la vez, asuman la importancia de contar con un conjunto de principios que asignen derechos, deberes básicos, el modo de la distribución correcta de las cargas y beneficios de la cooperación social. Es decir, aceptan la diferencia entre el concepto y las diversas concepciones de la justicia que cada uno pueda adscribir.

Pero no cede frente a lo que cree fundamental, el establecer un criterio de acuerdo acerca de lo justo e injusto con el fin de que las personas puedan coordinar sus planes y acciones en función del respeto de acuerdos que benefician a las partes, de lo contrario se corre el riesgo de que las acciones de las personas desgasten los vínculos del civismo, "La desconfianza y el resentimiento corroen los vínculos del civismo, y la sospecha y la hostilidad tientan al hombre a actuar de maneras que de otro modo evitaría” (Rawls; idem:23). ${ }^{1}$

Explicita que cualquier teoría ética relativamente completa debe incluir principios para definir la distribución de las instituciones básicas de la sociedad, y estos principios -independientemente de cuales sean- constituyen la doctrina de justicia.

\section{La Justicia como Imparcialidad: el velo de la ignorancia y la posición original}

Su preocupación ahora será mostrar el mecanismo por el cual se llegan a elegir dos principios que darán cuenta de su teoría de la justicia como imparcialidad o equidad. Ya sabemos que los principios regulan los derechos, deberes, los beneficios y los tipos de cooperación social aceptados en la estructura básica de la sociedad, ahora cuales principios serían elegibles y deseables, pero desde un punto de vista imparcial, realizado por personas racionales y libres es lo que pretende responder Rawls. La justicia como imparcialidad posee dos componentes, el primero es la posición inicial u original de los sujetos y la elección que realizarán acerca del proble-

\footnotetext{
${ }^{1}$ Esta afirmación nos recuerda a Hobbes y su concepción negativa acerca de la naturaleza del hombre por lo que se precisa establecer un contrato social con el fin de sacar al hombre del estado de naturaleza siempre en potencia de guerra y lucha de todos contra todos y depositar en un tercero la seguridad de la vida.
} 
ma a tratar y un segundo componente es el conjunto de principios acerca de los cuales habrá cierto acuerdo.

Centrándonos en el primer componente de la justicia como imparcialidad, podemos decir que Rawls llama la atención acerca de la existencia de unas “circunstancias de la justicia” que las entenderá como las condiciones normales bajo las que es posible y necesaria la cooperación humana, es decir, lo que ya previamente habíamos mencionado acerca de la coexistencia de identidad y conflicto de intereses entre los sujetos, pero donde prima la necesidad de cooperación social.

Profundizando en las circunstancias de la justicia menciona la existencia de condiciones objetivas y subjetivas que hacen posible y necesaria la cooperación humana -idea que reconoce ya presente en los escritos de Hume. Dichas condiciones objetivas las vincula a la semejanza en capacidades físicas y mentales existente entre los sujetos, la vulnerabilidad a la que pueden ser todos expuestos, como también la escasez moderada de recursos naturales y no naturales con que se cuenta en una sociedad.

Por otro lado, las circunstancias subjetivas las remite a que los sujetos de la cooperación poseen planes, concepciones y propósitos diferentes de vida, incluso conflictivos en muchos aspectos, pero el punto común entre todos ellos es que pretenden obtener del acuerdo, el máximo posible de ciertos bienes buscados por todos los participantes, ejemplo de ello son derechos, libertades, ingresos y riquezas, autorrespeto, oportunidades y poderes. Cree él que sin la existencia de estas circunstancias -la necesidad de cooperación y pluralidad con posibles conflictos de intereses- no es posible "la virtud de la justicia”. En la posición original en que se encuentran los sujetos, éstos asumen esas circunstancias aceptándolas como algo dado naturalmente o parte constitutiva de la sociedad.

La posición original equivale a establecer un cierto procedimiento equitativo -por lo que se le llama justicia como equidad-mediante el cual se elegirán unánimemente ciertos principios por los miembros de las sociedad -que independiente de quienes elijan y cuales sean los principios elegidos- serán asumidos porque se ha arribado a ellos siguiendo un determinado método. Rawls mostrará que los principios elegidos en esta posición original no podrían ser otros más que a los que él ha llegado.

Pero antes de avanzar en su demostración, el supuesto con el que cuenta es con la existencia de igualdad entre los sujetos, en tanto personas morales o como seres que poseen una concepción de lo que es bueno para ellas, y también capaces de tener un sentido de la justicia y una capacidad de actuar conforme a cualquiera de los principios de justicia adoptados.

Los sujetos en esta posición original participan de una situación ficticia, menciona que se encuentran cubiertos por un "velo de ignorancia" acerca de las características de los que participan en ella. Con esta fórmula Rawls quiere asegurar que los principios de justicia se escogerán en la más 
plena ignorancia, para asegurar a nadie posiciones de ventajas o desventajas producto de la fortuna natural o por las circunstancias sociales en que han vivido los sujetos. Tal procedimiento fija y garantiza que los acuerdos alcanzados respecto de la elección de los principios de justicia sean imparciales, a la vez que se permite que todos tengan los mismos derechos de elegir principios², "nadie sabe cuál es su lugar en la sociedad, su posición, clase o status social; nadie conoce tampoco cuál es su suerte con respecto a la distribución de ventajas y capacidades naturales, su inteligencia, su fortaleza, etc. Supondré, incluso, que los propios miembros del grupo no conocen sus concepciones acerca del bien, ni sus tendencias psicológicas especiales. Los principios de justicia se escogen tras un velo de ignorancia (...) si un hombre sabe que él es rico, puede encontrar racional el proponer que diversos impuestos sobre medios de bienestar sean declarados injustos; si supiera que era pobre, es muy probable que propusiera lo contrario. Para presentar las restricciones deseadas uno se imagina una situación en la que todos estén desprovistos de esta clase de información” (Rawls; idem: 29-36).

Incorpora la figura del contrato social donde -hipotéticamente- el estado de naturaleza se corresponde con la posición original de igualdad en que los participantes se ubican y relacionan bajo el velo de ignorancia. Si bien considera que la noción de contrato o acuerdo aplicado a materias morales puede llevar a equívocos e invitar a objeciones, plantea que esta noción no debe entenderse como el ingreso a un tipo de sociedad o adoptar un cierto tipo de gobierno -propuesto en la tradición contractualista de Hobbes, Locke, Kant, Rousseau- sino en adoptar ciertos principios elegidos en una situación inicial claramente definida. La figura del contrato ${ }^{3}$ -menciona- sugiere la pluralidad de relaciones entre personas que participan de la cooperación social, de sus diversos y conflictivos intereses, como del requisito de que la división correcta de ventajas tiene que hacerse acorde a principios aceptables y públicos para todas las partes.

Explicita ciertas restricciones formales del acuerdo de principios de justicia a que arriben las personas en la posición original. Entre ellas muestra cinco restricciones: primero, los principios deberán ser generales, es decir, la formulación expresarán relaciones y propiedades generales que

\footnotetext{
${ }^{2}$ La ignorancia no es absoluta, Rawls considera algunos conocimientos básicos que poseerán las partes entre los que encontramos los siguientes: conocen hechos generales de a sociedad humana, entienden las cuestiones políticas, los principios de teoría económica, las bases de la organización social y las leyes de la psicología humana.

${ }^{3}$ La teoría del contrato social desemboca en una concepción del orden político como limitación de la libertad de cada persona, pero a la vez subyace una noción individualista como punto de partida, es decir, es la persona quien determina todo el procedimiento de elección cuyo método es el "constructivismo kantiano". Lo anterior se traduce en que los principios de la ética serán creados por sus destinatarios, los cuales se suponen personas libres e iguales que al seguir ciertos procedimientos racionales construirán y aceptarán unos principios de justicia sin referencia a objetos morales cognoscibles o en deuda a una realidad externa al sujeto. Para mayor detalle, ver Massini, Carlos; El constructivismo de Rawls. Estudios Jurídicos. Universidad de Navarra.1997.
} 
no permita a las partes disponer de información específica acerca de sí mismos y de su situación, para evitar identificarse. Segundo, deben ser universales en su aplicación o valer para todos por ser estas personas morales. Una tercera condición es el carácter público, en el sentido de que las partes suponen que están escogiendo para una concepción pública de la justicia, es decir, debe ser reconocida públicamente como constitutiva de la vida social. La cuarta restricción es que la concepción de lo justo debe imponer una ordenación de las demandas conflictivas que puedan surgir. Por último, la quinta condición es la definitividad de su carácter justificatorio; las partes han de considerar el sistema de principios como el tribunal supremo de apelación en materia de razonamiento práctico. Avanza a la vez hacia la necesidad de justificar la elección de una concepción de justicia por sobre otra y esta justificación la vincula con la teoría de la elección racional, ya que parece razonable aceptar que en. La racionalidad la significa como aquel individuo que si bien posee sus preferencias, es capaz de jerarquizar las opciones en función de aquellas que mejor le permiten conseguir sus propósitos.

Supone una racionalidad de la que participan los individuos, y entre sus características menciona que a un "individuo racional no le asalta la envidia” puesto que este sentimiento hace peores a los sujetos, así mismo, su no existencia -de envidia- implica comprender que cada persona posee un plan de vida propio que le es suficiente y que no está dispuesto a abandonar. Tal racionalidad mutuamente desinteresada posee una interpretación en vías paralelas, una de ellas es aquella en que los sujetos están dispuestos a ganar la mayor cantidad de bienes sociales para sí, y la segunda es que en esta ganancia no pretenden dañarse entre todos -ya que no los mueve el rencor- ni beneficiarse de los demás. Otro rasgo de la racionalidad que subyace a su propuesta, la muestra en que las partes no están dispuestas a suscribir acuerdos que no se puedan cumplir o se encuentren con innumerables dificultades para hacerlo, a su vez afirma que al hacer sus propuestas, los sujetos no cuentan con el incentivo de sugerir principios insensatos o arbitrarios, ya que los principios se muestran como una limitación a la libertad de acción y el aceptar tales restricciones solo sería mediante una acción racional.

\section{Los Principios}

El primer principio exige la igualdad en la repartición de derechos y deberes básicos y el segundo principio mantiene las desigualdades sociales y económicas siempre y cuando éstas sólo producen beneficios compensadores para todos y especialmente para los sujetos menos aventajados de la sociedad, es decir, este segundo principio implica aceptar que unos pocos obtengan mayores beneficios con el fin de que se mejore la situación de los sujetos menos afortunados a diferencia de la lógica común en donde se justifica que unos pocos sufran privaciones si es que se compensa con que se obtenga un mayor bien para todos en general. 
Ambos principios los formula de la siguiente manera:

- "Cada persona ha de tener un derecho igual al más amplio sistema total de libertades básicas, compatible con un sistema similar de libertad para todos"

- "Las desigualdades económicas y sociales han de ser estructuradas de manera que sean para:

a) mayor beneficio de los menos aventajados, de acuerdo con un principio de ahorro justo, y

b) unido a que los cargos y las funciones sean asequibles a todos, bajo condiciones de justa igualdad de oportunidades” (Rawls; 1995: 341).

El primer principio refiere a todo tipo de libertades básicas -derecho a voto, desempeñar puestos públicos, de expresión, de reunión, de conciencia, de pensamiento, personal o de opresión psicológica, de agresión física, de propiedad personal, son algunos ejemplos-, el segundo se aplica a las desigualdades económicas y sociales, como la distribución del ingreso y la riqueza, al diseño de organizaciones que hagan uso de las diferencias de autoridad y responsabilidad (Rawls, idem.). Los bienes sociales primarios a distribuir son: derechos y libertades básicas, la libertad de circulación y la libre elección de ocupación en un contexto de oportunidades diversas, poderes y prerrogativas de cargos y posiciones de responsabilidad en las instituciones políticas y económicas de la estructura básica y renta y riqueza.

Los principios -observa- pueden ser interpretados “democráticamente” y con esa intensión remite a las tradicionales ideas de libertad, igualdad y fraternidad, ajustando la idea de libertad e igualdad al primer principio aunque la igualdad también concuerda con la justa igualdad de oportunidades- y la fraternidad al principio de la diferencia.

Pero no queda todo allí, incorpora un orden y unidad entre estos dos principios, lo cual se manifiesta en un "orden serial” o en la prioridad lexicográfica de uno sobre el otro, es decir, el primer principio posee prioridad sobre el segundo. Así también es prioritaria la segunda parte del segundo principio, la igualdad de oportunidades, sobre la primera parte del mismo. El orden lexicográfico, significa que hasta que no se consiga el nivel adecuado en uno de los principios, el siguiente no entra en juego. Rawls, con este orden hace evidente la distinción entre derechos y libertades fundamentales, y beneficios sociales y económicos, generando con ello la jerarquización de diversos bienes primarios, en donde las libertades básicas pasan a ser prioritarias e irrenunciables a pesar de que su abandono pueda compensar desde el punto de vista de las ventajas económicas y sociales. Especialmente en este punto se observa con gran nitidez su arraigo liberal.

Plantea que este ordenamiento de los principios se configura casi intuitivamente entre las partes y en contraposición al principio de utilidad, 
ya que los sujetos en la posición original se entienden primeramente como personas libres que poseen intereses y objetivos fundamentales en nombre de los cuales creen legítimo hacerse demandas mutuas en función de la confirmación de la estructura básica de la sociedad. Mediante tal ordenamiento cree que todos los intereses fundamentales de las partes están garantizados en la medida que se anteponga el principio de libertad por sobre el segundo. Pero esa prioridad que le otorga a la libertad es casi absoluta puesto que abre la opción de negar o restringir la libertad siempre y cuando con esa acción se logre cambiar las condiciones de la civilización, de manera de permitir en un tiempo determinado gozar nuevamente de tales libertades.

\section{La Estrategia Maximin como Elección Conservadora}

El principio denominado "libertad equitativa” es el "máximo natural” del primer principio y el principio llamado de “diferencia” es el “máximo natural” del segundo principio, por lo que Rawls asumirá que estos dos principios son la solución óptima elegida por las personas en la posición original. Concibe que la elección de ellos, son una especie de solución máxima porque siempre los peores resultados serán superiores al (los) peor(es) resultado(s) de cualquier otra. Los dos principios son pensados por este autor, como la solución maximin al problema de la justicia. Lo que distingue a la regla maximin es que se define como una estrategia alternativa a otras dos opciones disponibles; la maximax y la maximización de la utilidad esperada. La alternativa maximax refiere a que se debe ordenar las alternativas por sus mejores resultados posibles, adoptando aquella cuyo mejor resultado sea mejor que el mejor resultado de las demás, así se muestra como una estrategia para optimistas. La alternativa de la maximización se plantea el ordenamiento de las alternativas sobre la base de estimaciones de ganancia probable.

Rawls rechaza la estrategia maximax por presentarse altamente arriesgada ya que las posibilidades de perder son grandes. También rechaza la segunda estrategia porque en la posición original, en las que el conocimiento de probabilidades es imposible o inseguro, se precisa ser escépticos a propósito de los cálculos, ya que el velo de la ignorancia no permite tener claridad acerca de la probabilidad de obtener ganancias.

La regla maximin permite ordenar las opciones por los peores resultados posibles que ofrecen y se aceptará aquella cuyo peor resultado sea superior a los peores resultados de las otras. La regla maximin es una estrategia que se justifica y que resultaría atractiva a alguien con una actitud conservadora o pesimista, pero a propósito de la situación especial de la posición original ya que surge la duda de que esta sea la mejor alternativa en la vida real. Las partes eligen principios para el diseño de una sociedad como si los lugares que fueran a ocupar en ella fueran determinados por sus peores enemigos. 


\section{La Justicia Materializada en las Instituciones}

Para superar la forma abstracta de esta concepción de justicia, Rawls apunta en la segunda parte de su libro, a mostrar las implicancias substantivas que se derivan de adoptar estos principios (cuyas nociones de libertad, oportunidad, equidad aun vagas y ambiguas) a nivel de las instituciones. En ese despliegue intenta clarificar la significación y consecuencias prácticas de ambos principios lo cual ilustra describiendo la estructura básica que los satisface, estructura de la cual fluyen ciertas instituciones o prácticas básicas, al interior del marco de una democracia constitucional y liberal. Una constitución política justa, arreglos económicos justos y obligaciones de las personas de obedecer las leyes de una sociedad que es imperfectamente justa serán ejemplos de eso.

.El vínculo que establece Rawls entre los principios de justicia elegidos en la posición original y las instituciones justas, lo va aclarando paulatinamente, y en una especie de ejercicio metódico pide imaginar una secuencia de acontecimientos en cuatro estadios o pasos que -en su conjunto- darán cuenta de esa vinculación. El primer paso describe la elección de los principios bajo el velo de la ignorancia, el segundo está destinado a que las partes que participan de la posición original se reúnan y decidan sobre la justicia de las formas políticas, como también elijan una constitución, en el que se aclaran los derechos y libertades básicos, el tercer paso está destinado a establecer leyes -legislar- que afecten a la estructura económica y social de la sociedad y, por último, en el cuarto ocurre la aplicación de las reglas por parte de los jueces y demás funcionarios, previamente dado una comprensión plena de la estructura básica -y justa- de la sociedad.

El sistema político justo en este ámbito lo será siempre que se incorporen y protejan las libertades de la ciudadanía al interior de la constitución política. La libertad nunca puede ser negada o restringida en la teoría y la instancia que materializa esta idea es una constitución justa que deberá entender y proteger la libertad, que incluye libertad de conciencia, pensamiento, personal y la igualdad en los derechos políticos. Es claro en declarar que el sistema político acorde con ello sería alguna forma de democracia constitucional, pero da cuenta de la limitación de alcanzar un estado ideal de justicia por vía de una constitución en la sociedad concreta, por lo que lo mejor a que se puede aspirar es una justicia procesal imperfecta. Pero a pesar de eso, cree firmemente en que en la medida que se defina el campo de la justicia ideal se permitirá evaluar las soluciones existentes y se identificarán con mayor nitidez los problemas y dificultades más graves que una sociedad debe evitar o los cambios que debe impulsar.

Las funciones de asignación, de estabilización, de transferencia y de distribución, serán ejercidas por el gobierno para dar cuenta de lo anterior. En ese sentido, se logra visualizar que la justicia económica expresada por el segundo principio de justicia se materializaría mediante los órganos de 
un gobierno, instancia más cercana a la figura que algunos comentaristas han vinculado a un Estado de Bienestar ${ }^{4}$ que a la del Estado mínimo.

\section{Algunas observaciones a la Teoría de la Justicia}

En estos años ha sido inmensa la producción teórica que en algún modo critica o aprueba la Teoría de la Justicia, a continuación mostraremos algunas críticas que se han levantado a esta propuesta, de modo de mostrar algunos de los límites que posee.

Siguiendo a Vallespín (1998) es claro que Rawls muestra una confianza en poder sustentar una concepción pública de la justicia, válida para las sociedades contemporáneas donde la pluralidad de modos de vida cuestiona la aceptación de una única concepción del bien que rija para todos, por lo mismo, la construcción rawlsiana representa la creencia en la razónracionalidad como vía -aún capaz- de construir ciertos procedimientos de argumentación racional que logren sintonizar esa pluralidad, más allá del “carácter finito y falibilista de la razón, ciertamente reducida en su capacidad para poner orden” (Vallespín; 1998: 13). Tal edificación se sostiene sobre "delgados pilares", como es la coherencia interna de los principios y la aceptación por parte de los que participan de la posición original, argumentación que deja de lado cualquier concepción de objetividad vinculada a la verdad como correspondencia con la estructura de la realidad o una verdad gestada históricamente.

El acento en estos elementos procedimentales favorecerían la prioridad y distinción de la justicia sobre el bien, es decir, diferencia entre la justicia y lo que es bueno para un cierto grupo en un determinado contexto, definición contextual que Rawls ve imposible de universalizar. Pero algunos críticos evidenciaron la inconsistencia de ello en la misma obra ya que Rawls acaba aceptando algunas nociones de bien humano que permiten darle sentido a la organización social, a través de la definición de bienes primarios válidos para todos y "suponiendo -aunque sea sin aceptarlo explícitamente- toda una concepción de la vida buena o perfección humana social: una vida presidida por la autonomía de los sujetos y la tolerancia de y hacia los demás, de vigencia de los derechos humanos, de participación política en democracia y así sucesivamente” (MacIntire, en Massini; 2005: 183). Este elemento de crítica será superado en su obra Liberalismo Político, en donde ajusta cuentas con esa observación.

Otro orden de objeciones deriva del énfasis puesto en una ética de las estructuras institucionales, invisibilizando la dimensión de la ética humana que conlleva sus propias motivaciones, deseos y fines. Considerar

\footnotetext{
${ }^{4}$ Este punto es un tanto obscuro en esta obra, ya que en distintos pasajes se refiere o a una democracia constitucional, en otros a una democracia de particulares y en otros a una democracia liberal.
} 
este elemento obliga a preguntarse por la posibilidad de incidencia que posee ello una vez levantado el velo de la posición original, la inseguridad de que los sujetos respeten el acuerdo realizado una vez conocidas sus propias motivaciones morales parece ser acertado. La crítica de Walzer al respecto se puede resumir en que difícilmente los sujetos racionales que eligen en condiciones de imparcialidad, llevados de vuelta a gente común, con un sentido de la propia identidad e inmersos en los problemas cotidianos, van a reiterar su "hipotética elección”.

Rawls, al rechazar que las particularidades de la historia, la cultura y la pertenencia a un grupo definan la elección de los principios de la justicia, puntualiza su construcción como universalista y abstracta, lo cual deja de lado las diferencias, la "otredad", materializadas en las múltiples minorías que hoy claman sus derechos a participar de las decisiones. En este punto, la teoría rawlsiana de la justicia no da cuenta de la dimensión de las identidades, el género y del reconocimiento. Iris Marion Young (2000) objeta le centralidad de la distribución en Rawls porque no considera los temas de opresión y dominación ni cuestiona las estructuras sociales ni los contextos como las instituciones capitalistas y las relaciones de clase.

El principio de diferencia (Massini, 2005; Gerald Cohen, 2001) se constituye en un principio que posibilita el conformismo con el liberalismo, y una inmensa desigualdad siempre y cuando ambas partes reciban algún beneficio. A partir del principio maximin se argumenta que los más desfavorecidos deben aceptar las ventajas que se les brindan, ya que de otro modo se encontrarían en una situación peor. Una regla explicitada en esos términos anula cualquier intensión de transformación radical de las estructuras sociales que mantienen las desigualdades. La predilección por el cumplimiento de ciertos derechos los torna una estructura cerrada, inmutable frente al movimiento y luchas de las distintas formas de vida por alcanzar la igualdad social-económica y el reconocimiento de la identidad.

Desde el mismo campo de batalla, Sen y Habermas apuntan su fuego amigo. Habermas (1998) llama la atención de la dificultad en que se encuentra Rawls al asimilar los bienes básicos a derechos, bienes generalizados que los sujetos pueden necesitar para realizar sus planes de vida. Ello implica que los principios de justicia se reduzcan a la justa distribución de los bienes básicos, más que a la autonomía de los sujetos a través del ejercicio de los derechos. Habermas también se opone al diseño de la posición original en la que la elección se basa en sujetos "egoístas racionales”, pone en duda que la imparcialidad se logre mediante la reflexión individual donde priman generalmente intereses egoístas. Amartya Sen, por su lado, menciona acerca del riesgo fetichista que se corre al asumir la centralidad de los bienes primarios o recursos a la hora de hacer comparaciones interpersonales. Interpreta esos bienes primarios como un equivalente a los medios para la libertad para propósitos generales, pero insiste en que si el eje de preocupación es la libertad, no es suficiente centrarse en los medios para la libertad sino en la amplitud de la libertad que realmente tiene la persona debido a que "la capacidad para convertir estos bienes 
primarios y recursos en libertad -para seleccionar una vida particular y para alcanzarla- puede variar de persona a persona, la igualdad en las posesiones de bienes o las libertades reales de que gozan las diferentes personas” (Sen, Amartya; 1998: 115).

Por lo tanto, la evaluación de la justicia basada en capacidades, las demandas individuales no se valoran en términos de los recursos o bienes primarios que poseen las diversas personas, sino en términos de las libertades de que realmente gozan para elegir entre los diferentes modos de vivir que pueden tener razones para valorar, es "la libertad real la que representa la “capacidad” de una persona para conseguir las varias combinaciones alternativas de realizaciones, esto es, de haceres y estares”. La posesión de bienes primarios o recursos no representa la capacidad de la que goza una persona $^{5}$.

\section{Los retos de la Justicia social frente al contexto actual}

La filosofía griega nos muestra esa maravillosa relación entre justicia, sociedad, comunidad y ciudadano como parte del hacer en y de la polis, a cuya grandeza Jaeger nos sumerge y nos invita a reconocer. Esta relación -y la prioridad de la justicia- en los orígenes del pensamiento occidental se presenta desde los poemas épicos de Homero como también en los primeros filósofos griegos quienes entendieron la justicia como el centro de la cultura humana y la clave para dar cuenta del lugar del hombre en el cosmos (Jaeger, 1982) ${ }^{6}$. Es decir, la justicia, era parte y a la vez permitía un orden cósmico, una armonía y jerarquía de las acciones humanas. Este orden y armonía en la ciudad, construido a través del ejercicio ciudadano de la política en la esfera pública también sirvió para naturalizar diferencias entre hombres-ciudadanos y esclavos, mujeres, niños, extranjeros y ancianos.

\footnotetext{
${ }^{5}$ Sen cita algunos ejemplos que clarifican dicha afirmación, en pobreza menciona que "al considerar la pobreza en los países ricos, tenemos que tener en cuenta el hecho de que muchas de las personas que son pobres en términos de renta y de otros bienes primarios también tienen características -edad, incapacidades, propensión a las enfermedades, etc.que hacen más difícil para ellas convertir bienes primarios en capacidades básicas, por ejemplo, la capacidad de desplazarse, para llevar una vida sana o para tomar parte en la vida social” (Sen, Amartya, 1998:115)

6 "En el pensamiento homérico, pues, era dique la línea de demarcación entre la barbarie y la civilización. Dondequiera que la justicia impere, pisa el hombre tierra firme, gozando de seguridad y protección en su persona y sus bienes, a salvo incluso como peregrino en tierra extraña”, o cuando refiere a Heráclito y Anaximandro: "Heráclito, pues, no solo concibió el principio del universo como una ley o una justicia de índole cósmica, a la manera de Anaximandro, sino que conectó, además, directamente la ley humana y la vida del hombre en comunidad con aquel orden divino. Ello implicaba que todo ser individual desempeña su papel en el mundo, como todo ciudadano de una polis griega desempeña el suyo en la vida constitucional de su comunidad” (Jaeger, 1982: 8, 32).
} 
El cuestionamiento acerca de la naturalización de la desigualdad social y política lo apunta brillantemente Rousseau, quien plantea que ésta no deriva de la voluntad divina, ni es consecuencia de la desigualdad natural entre los hombres, al contrario su origen es resultado de la propiedad privada de la riqueza y de los beneficios que ese ejercicio otorga ${ }^{7}$. El tratar de explicar el origen de la desigualdad social como la legitimidad de que es objeto, nos lleva a centrar la atención en la estructura económica, social, política y normativa que permite aquello. Fenómenos contemporáneos cristalizan la cara sórdida de aquel imaginario griego, pero, a la vez ¿̇no expresan un desafío para la conceptualización y materialización de la justicia social?

Como sabemos, las guerras de las últimas décadas muestran un parámetro distinto de funcionamiento que las realizadas hasta la primera mitad del siglo XX, hoy las guerras adquieren una forma monstruosa para la sociedad civil, donde la cantidad de muertos civiles -especialmente mujeres, niños y ancianos- es mucho mayor que las bajas de las fuerzas militares de los bandos en pugna, a su vez, los sobrevivientes muchas veces se transforman en desplazados, hombres y mujeres que recorren el país evitando encontrarse en el fuego cruzado, el refugio en muchos casos ha tendido a disminuir porque los países aledaños al conflicto tienden a cerrar sus fronteras. A lo anterior se deben sumar las muertes indirectas causadas por enfermedades o hambrunas producto de la guerra. Baste decir que el trasfondo de la mayoría de esas guerras son intereses económicos y de dominación, para comprender que la semántica de las "guerras justas" cumple una función de encubrimiento y justificación.

Por otro lado, la declaración del milenio derivada de la Asamblea General de las Naciones Unidas el año 2000, criticaba las enormes desigualdades en el desarrollo humano de los países e impuso la tarea de reducir la pobreza a la mitad al año 2015, pero esa intensión no deja de ser una ficción. Las cifras hablan por si solas, hasta el 2007 -según el informe de evaluación y monitoreo del PNUD- existían 980 millones de personas en los países en desarrollo que sobrevivían con menos de un dólar diario, cifra que hoy se ha elevado a 1.400 millones según la nueva medición de pobreza realizada por el Banco Mundial, que da cuenta de nuevas 400 millones de personas que viven por debajo de la línea de pobreza, ubicada ahora en un dólar y veinte y cinco diariamente en los países en desarrollo. Las mismas estimaciones del Banco Mundial mencionan que al año 2015 serán 1.000 millones de personas las que estarán en situación bajo la línea de

\footnotetext{
7“El primero que, habiendo cercado un terreno, descubrió la manera de decir: Esto me pertenece, y halló gentes bastante sencilla para creerle, fue el verdadero fundador de la sociedad civil. ¡Qué de crímenes, de guerras, de asesinatos, de miserias y de horrores no hubiese ahorrado al género humano el que, arrancando las estacas o llenando la zanja, hubiese gritado a sus semejantes:"Guardaos de escuchar a este impostor; estáis perdidos si olvidáis que los frutos pertenecen a todos y que la tierra no es de nadie"i(Rousseau; 1999: 60).
} 
pobreza, y muchos de los que han superado el dólar y veinte y cinco, diario seguirán siendo pobres ${ }^{8}$, situación que desacredita los compromisos del milenio.

Lo anterior es acompañado por un aumento en la desigualdad de ingresos, la existencia de 800 millones de desnutridos en el mundo, 2.600 millones de personas sin acceso a un saneamiento básico, 1.8 millones de niños muertos al año como consecuencias de diarreas y enfermedades derivadas de agua contaminada o saneamiento insuficiente (PNUD, 2006). Alrededor de 50.000 seres humanos mueren diariamente (especialmente niños y personas de color), ya sea de hambre o enfermedades como diarrea, neumonía, malaria y otras causas relacionadas con la pobreza. (Pogge, Thomas; 2007).

La concentración de la riqueza es escandalosa ${ }^{9}$, según el X informe sobre la riqueza mundial de Merril Lynch y Capgemini del 2006, el número total de millonarios en el mundo creció en un $6.5 \%$ en el año 2005, alcanzando unos 8,7 millones de personas -correspondiente al 0,1\% de la población mundial- que controlan 33,3\% billones de dólares y al 2010 se estima que el patrimonio en manos de los millonarios alcanzará 44,6 billones de dólares. Incluso en situación de crisis como la que viene sucediendo desde mediados del año 2007, desatada por el mercado inmobiliario, un estudio estadounidense del año 2008, a cargo de The Boston Consulting Group (BCG) muestra que la acumulación o aumento de la riqueza ha sido sostenido en los últimos seis años, así los que eran ricos no dejan de ser ricos y a la vez se suman los nuevos ${ }^{10}$, que se

\footnotetext{
${ }^{8}$ Para mayor detalle ver sitio del Banco Mundial en: http://go.worldbank.org/MLVZFZTMS0

${ }^{9}$ Para detalles de la concentración mundial, Ver www.iarnoticias.com o Universidad de las Naciones Unidas UNU-WIDER, en www.wider.unu.edu/ . Los análisis dan cuenta de una estructura integrada de desigualdad en las economías avanzadas como EEUU e Inglaterra en los años 90, en que la brecha entre ricos y pobres en función de los ingresos ha aumentado como también dificultad de movilidad social. (Callinicos, Alex; 2003). Algunos datos en Chile muestran la tendencia de la concentración del capital, así tenemos que la distancia de ingresos entre el 5\% más rico y el 5\% más pobre aumentó entre los años 1990 y 2005, pasando de 110 a 220 veces, creciente polarización que no tiene que ver con talentos naturales, ni trabajo o sacrificios. Desde el año 2003 al 2007 las compañías multinacionales se llevaron casi el 50\% del PIB (Cademartori, José; 2008), por otro lado la concentración de la riqueza en Chile se acumula en tres grupos económicos cuyas ganancias aumentan año a año; tenemos así que el ranking lo encabeza el grupo Luksic que de 3.400 millones de dólares poseídos al año 2004 aumentó a 4.200 en el año 2005, le sigue el grupo Angelini que de 2.500 millones de dólares al 2004 aumentó a 2.900 en el 2005 y por último el grupo Matte que de 2.800 millones de dólares al año 2004 retrocedió a 2.700 en el año 2005. (Claude, Marcel; 2008)

${ }^{10}$ El periódico español El País, en su columna en economía llama la atención sobre este fenómeno, el crecimiento de las nuevas familia millonarias, el cual alcanzó un 11\%, especialmente por los aportes de familias provenientes de Asia y América Latina, así también muestra como aquellas familia que ya son millonarias se las arreglan para aumentar su crecimiento de fortunas a pesar de la crisis hipotecaria. Ver Diario El País, economía, 5/9/ 2008, en http:/www.elpais.com/articulo/economia/ricos/vez/ricos/pesar/crisis/financiera/ elpepueco/20080905elpepueco_6/Tes
} 
las arreglan haciendo los ajustes necesarios, en el contexto de la crisis financiera, para nunca perder.

La destrucción ambiental, trae sus propias consecuencias a nivel mundial, afectan y lo harán -a futuro con mayor gravedad- desigualmente a los países y al interior de estos aumentando la pobreza ${ }^{11}$. En el marco del capitalismo, los recursos naturales se tornan cada vez más en capital financiero en beneficio de los grupos económicos y en detrimento de los recursos naturales. La creciente deforestación, degradación de tierras secas (sin contar los desiertos), el riesgo de extinción y desaparición de especies ha ido en aumento, la escasez y restricciones de acceso a agua se vaticina para las dos terceras partes de la población mundial en los próximos 25 años son solo algunos de los efectos de esta masacre ambiental (Claude, M.; 2007: 10). A ello debemos incluir el informe sobre derechos humanos de Amnistía Internacional (2007) que relaciona la búsqueda de recursos naturales por parte de grandes conglomerados económicos con la persistente amenaza de la identidad cultural y supervivencia de varias comunidades indígenas en América Latina.

Un fenómeno de no larga data en los países, especialmente de Europa y América del Norte, se relaciona con la "seguridad frente al terrorismo", idea que se ha vuelto capaz de justificar acciones paranoicas que derivan en discriminación, control, detenciones injustificadas, violación del derecho de libertad, etc. (Amnistía Internacional; 2007). Desde otro frente, Kurz (1997), menciona que los fundamentalismos de orden (pseudo)religiosos vienen a constituirse en formas de castigo merecido, por la soberbia de la economía de mercado, del sistema capitalista que impone un modo de vivir unilateral con consecuencias de desintegración brutales.

Otra dimensión -con el mismo sustrato- vemos en la legitimación de la criminalización de los inmigrantes ilegales por parte de la unión europea, que refleja un resabio xenofóbico, de racismo y discriminación contra millones de personas que ven en los países centrales una salida a la permanente situación de pobreza y desigualdad que viven en sus países de origen. La paradoja de todo esto es que la función del Estado se torna mínima y máxima a la vez, la primera materializa las inacciones de regulación o "libertad” de mercado y la segunda, una máxima intervención policial y leyes punitivas. Marx, en La Cuestión Judaica de 1843 advertía brillantemente sobre este último punto al declarar "La seguridad es el concepto social

\footnotetext{
${ }^{11}$ El Pnud habla de que a medida que avanza el cambio climático, esto traerá mayores retrocesos a escala global. "Los reveses en el desarrollo humano no serán lineales, y sus poderosos efectos se retroalimentarán mutuamente. Las pérdidas en la productividad agrícola reducirán los ingresos, disminuirán el acceso a la salud y a la educación. A su vez, menores posibilidades detener acceso a la salud y a la educación restringirán las oportunidades de mercado y reforzarán la pobreza. En un nivel más fundamental, el cambio climático dañará la capacidad de las personas más vulnerables del mundo de tomar decisiones y perfilar procesos que tengan efectos en su propia vida” (PNUD, 2007: 107).
} 
supremo da la sociedad burguesa, el concepto de policía, según el cual toda la sociedad existe solamente para garantizar a cada uno de sus miembros la conservación de su persona, de sus derechos y de su propiedad” (Marx, 2005: 37). Seguridad y libertad son caras de la misma moneda.

\section{Algunas Conclusiones: entre lo normativo y los fenómenos sociales}

¿Será posible combatir lo anterior con una redistribución de recursos?, ¿¿se puede terminar o reducir la injusticia social en una economía capitalista, donde la pobreza y la desigualdad son solo algunos ángulos de ésta?, difícilmente se puede afirmar que es posible. El capitalismo es constitutivamente inestable, oscila entre fases de expansión y depresión, "está sujeto de forma crónica a colapsos profundos y disruptivos en la producción y en el empleo” (Callinicos; 2003:137), además genera conductas que tienden hacia el hedonismo, el egoísmo, el consumo exacerbado, el individualismo, cuyas consecuencias nefastas no se expresan solo en la desigualdad de la riqueza como ya vimos.

Estas ideas son las primeras que aparecen si es que se descuida la importancia de la pregunta por la Justicia que declara el horizonte normativo y la buena sociedad que se desea construir. En ese aspecto esta pregunta trae inscrita dos dimensiones: valorar, enjuiciar el presente para mirar el futuro a partir de algunos elementos existentes en el presente que permitirían construirlo.

Es importante fijar la potencia que poseen los valores y principios que pueden guiar la futura sociedad. Ya Macpherson -desde la tradición marxista- hablaba de que Rawls podría ser llevado a cabo en una sociedad sin clases. Fernando Lizárraga (2009) sintetiza -adecuadamente- los motivos de la importancia de Rawls para las discusiones que se enfrentan en esta materia. Rawls rechaza el utilitarismo y sus medidas subjetivistas de bienestar, la meritocracia, la tesis de autopropiedad (cuyo representante es Nozick), además propone una medida objetiva para valorar la igualdad (los bienes sociales primarios), ensaya reglas de prioridad para evitar o disminuir la arbitrariedad de las intuiciones al momento de tomar decisiones morales, en este punto, la prioridad de la justicia sobre la eficiencia y de lo justo sobre lo bueno resulta contraria al sentido común de la época actual y al estilo del capitalismo en la fase neoliberal. Por último, es interesante de considerar ya que no excluyó la posibilidad de que los dos principios que definen lo que es la justicia (en su aparato conceptual) fueran posibles de llevar a cabo en una sociedad donde existe propiedad social de los medios de producción. Podríamos agregar que Rawls transparenta la injerencia que poseen las instituciones sociales en generar, fortalecer y potenciar las desigualdades sociales.

Sin duda que al examinar en detalle estos elementos vemos que las respuestas y propuestas de la Teoría de la Justicia no permiten la cristaliza- 
ción -como dice Gerald Cohen (2001) de la "Igualdad radical de oportunidades" que obliga a corregir todas las desigualdades originadas de vivir o nacer en condiciones sociales desventuradas y no escogidas. La historia muestra que cualquier intento de igualar esas condiciones bajo un modelo económico capitalista tendrá como consecuencias grandes movimientos y salidas de capitales u oposiciones de tal magnitud que solo dejan sangre en el país que lo intenta. La lógica del capital profundamente depredadora -en su fase actual- es incompatible con una sociedad igualitaria de bienes primarios y generación de capacidades, y muestra de ello lo entrega cada cierto tiempo cada Informe de Desarrollo Humano, que solo ratifica la incapacidad de materializar dichos principios.

A la vez, ante la pregunta por la posibilidad de reconciliación en sociedades en que no solo la diferenciación creciente de modos de vida o la pluralidad de concepciones morales y acerca del bien existentes, sino también se convive con innumerables formas de degradación y regresión humana, parece ser una pregunta que traspasa las respuestas y soluciones apuntadas por Rawls.

Al evaluar la propuesta de Rawls, no solo en función de la sociedad norteamericana sino a nivel global -ya que la regresión de derechos sociales traspasa el ámbito local- surge la inquietud acerca del modo de hacer converger este retroceso social con la elección de un cierto conjunto de principios de justicia como los propuestos por Rawls, esta concepción pública de la justicia, ¿ es sustentable en sociedades donde la pluralidad llega hasta la negación de la dignidad humana, o donde los que participan en la posición original no son todos los que efectivamente deben estar?, ¿cómo podrían participar aquellos que no han sido objeto de reconocimiento más que para ser desechados?. Sin duda que la redistribución de los bienes sociales y primarios propuestos por Rawls, en función de los desventajados, es absolutamente insuficiente para una sociedad como la nuestra. El no romper con las estructuras que causan la pobreza y la desigualdad, la intensión de mejorar el resultado de una situación injusta establecida históricamente no hace más que mantener su reproducción.

Por otro lado, la teoría de Rawls es ciega frente a la relación entre política y grandes consorcios que influyen -sin duda- en la concentración del poder económico y baste para eso recordar los casos de transnacionales como Shell en Nigeria donde se alía a la esfera política, militar para mantener sus intereses ${ }^{12}$. El predominio del mercado sobre el Estado es un hecho

\footnotetext{
${ }^{11}$ El Pnud habla de que a medida que avanza el cambio climático, esto traerá mayores retrocesos a escala global. "Los reveses en el desarrollo humano no serán lineales, y sus poderosos efectos se retroalimentarán mutuamente. Las pérdidas en la productividad agrícola reducirán los ingresos, disminuirán el acceso a la salud y a la educación. A su vez, menores posibilidades detener acceso a la salud y a la educación restringirán las oportunidades de mercado y reforzarán la pobreza. En un nivel más fundamental, el cambio climático dañará la capacidad de las personas más vulnerables del mundo de tomar decisiones y perfilar procesos que tengan efectos en su propia vida” (PNUD, 2007: 107).
} 
fundamental, Kurz acierta cuando menciona que "el Estado no posee ningún medio primario de regulación, mas bien depende del medio del mercado, esto es, del dinero. Entretanto el medio "poder" atribuido al Estado y, teóricamente, en la mayoría de las veces, identificado con el dinero no posee ningún grado jerárquico primario, a penas un grado secundario, pues todas las medidas del Estado precisan ser financiadas, no solamente las actividades jurídicas, infraestructurales, etc., sino también el poder en el sentido mas inmediato del término, o sea, las fuerzas armadas. En ese sentido, ni los militares son un efectivo "factor extraeconómico", pues ellos también están sometidos al medio mercado, a través del problema de su financiamiento" (Kurz; 1997: 103).

Tras el recorrido iniciado podemos decir que la justicia demanda algo más que la construcción de una mejor forma de distribución, sino una transformación real de las estructuras de producción y de distribución. La debilidad de la teoría de la justicia presentada es la creencia en que la justicia puede realizarse al interior del sistema capitalista, obviando -según Callinicos- el papel de la explotación en la creación y mantenimiento de las estructuras existentes de desigualdad como de todas las diversas formas de regresión humana.

La dimensión normativa de la justicia frente a los fenómenos empíricos e históricos que estamos viviendo, nos permite evaluar este presente como regresivo para la humanidad, de carrera hacia el abismo, sin una noción de justicia y de buena sociedad a la que aspiramos difícilmente estos fenómenos de regresión (y que nos sitúan al borde del colapso) se tornarían problemáticos y repudiables.

Los aportes de Rawls acerca de concebir una sociedad justa, nos inducen -sin que lo imagine- a pensar que ellos sólo pueden ser logrados como dice Callinicos- en contra del capitalismo, creemos que el desafío puesto a la concepción de la justicia social rawlsiana en el contexto capitalista, es el modo de mostrar los límites inherentes que posee para afrontar las consecuencias de este sistema hegemónico. Es urgente -ya lo vienen diciendo varios pensadores- imaginar otros mundos posibles, donde la sustentabilidad, la democracia, la justicia social y la vida digna sean su centro y para ello los valores y principios que inspiren esa sociedad son fundamentales para incubar ese futuro. 


\section{Bibliografia}

Anistía Internacional (2007), Informe 2007. El estado de los Derechos Humanos en el Mundo. Editorial Amnistía Internacional. Madrid. España. En http://report2007.amnesty.org/esl/Download-the-Report. Revisado el 30 de julio de 2008 .

Banco Mundial (2008), El Banco Mundial Actualiza Estimaciones de la Pobreza en el Mundo en Desarrollo. En http://go.worldbank.org/ MLVZFZTMS0. Revisado el 10 de septiembre de 2008.

Cademartori, José (2008), La Extrema Riqueza: Causa de las desigualdades en Chile. Crónica digital, Cenda, Santiago de Chile, 2008. Revisado el 27 de julio 2008.

Callinicos, Alex (2003), Igualdad. Editorial Siglo XXI. Madrid. España.

Claude, Marcel (2008), “El Retorno de Fausto. A propósito del gobierno de Lagos”. Periódico Mensual El Ciudadano, año IV, Nº 58. Julio. Santiago. Chile

Idem (2007), Manifiesto Eco-Socialista. Bases preliminares para fundar el eco-socialismo del siglo XXI en Chile. En http://www.rebelion.org/docs/ 60177.pdf . Revisado el 20 de Julio de 2008.

Cristi, Renato (2000), “Sociedad Civil y Estado en la Filosofía del Derecho de Hegel”. En Seminarios de Filosofía. Pontificia Universidad Católica de Chile, vols. 12-13, pp.147-162. Santiago. Chile.

Cohen, Gerald (2001), Si eres Igualitarista, ¿cómo es que eres tan rico? Ediciones Paidós. Barcelona. España.

Fazio, Hugo (2005), Mapa de la Extrema Riqueza al año 2005. Colección Ciencias Sociales. LOM Ediciones. Santiago, Chile.

Ferrater Mora, José (2004), Diccionario de Filosofía. Editorial Ariel. Barcelona, España. Tercera reimpresión, Primera edición 1994. Tomo I.

Gargarella, Roberto (1999), Las Teorías de la Justicia después de Rawls. Un breve manual de filosofía política.Editorial Paidós. Barcelona. España.

Kurz, Robert (1997), “A falta de autonomia do Estado e os limites da política: quatro teses sobre a crise da regulaçao política”. En Os últimos combates. Editora Vozes. Petrópolis. Brasil.

Kukathas, Chandran, Petit Philip (1990), La Teoría de la Justicia de John Rawls y sus críticos. Editorial Tecnos. Madrid. España.

Lizárraga, Fernando (2009), “El Marxismo frente a la utopía realista de 
Rawls”. Artículo presentado a la revista UNICAMP. Brasil. 2009. (pronto a publicar).

Locke, John (1990), Segundo Tratado del Gobierno Civil. Alianza Editorial, España.

Marx, Karl (2005), A Questao Judaica. Editora Centauro. Sao Paulo. Brasil.

Massini, Carlos (2005), Filosofía del derecho. La Justicia. Editorial Lexis Nexis. Buenos Aires. Argentina.

Habermas, Jürgen (1998), "Reconciliación Mediante el Uso Público de la Razón”. En Debate sobre el Liberalismo Político. Editorial Paidós. Barcelona, España

Jaeger, Werner (1982), Alabanza de la ley, Colección Civitas, Centro de Estudios Constitucionales. Madrid. España.

Perelman, Chaim (2005), Etica e Direito. Editorial Martin Fontes. Sao Paulo. Brasil.

Pogge, Thomas (2007), "Reconocidos y violados por la ley internacional: los derechos humanos de los pobres globales”. En Justicia Global, Derechos Humanos y Responsabilidad. Por Giusti Miguel y Cortés Francisco. Siglo del Hombre Editores. Bogotá. Colombia.

PNUD, (2007), Informe sobre Desarrollo Humano 2007-2008. La lucha contra el cambio climático: solidaridad frente a un mundo dividido. Nueva York. EEUU. Versión digital En www.hdr.undp.org/en/espanol/, revisado 15 de julio 2008.

PNUD, (2007), Informe sobre Desarrollo Humano 2006. Más allá de la escasez: poder, pobreza y la crisis mundial del agua. Nueva York. EEUU. Versión digital En www.hdr.undp.org/en/media/, revisado 15 de julio 2008. RAWLS, JOHN (1995); “Teoría de la Justicia”. Fondo de Cultura Económica. Madrid. España.

Rousseau, Jean (1999), El Origen de las Desigualdades entre los Hombres. Ediciones Universales. Bogotá, Colombia.

Salvat, Pablo (2002), El Porvenir de la Equidad. Aportaciones para un giro ético en la Filosofía Política Contemporánea. Ediciones LOM. Santiago. Chile.

Vallespín, Fernando (1985), Nuevas Teorías del Contrato Social: John Rawls, Robert Nozick y James Buchanan. Alianza Editorial, Madrid. España.

Idem (1998), “Una Disputa de Familia: El Debate Rawls-Habermas”. En Debate sobre el Liberalismo Político. Editorial Paidós. Barcelona, España. 